INTERNATIONAL DESIGN CONFERENCE - DESIGN 2018

https://doi.org/10.21278/idc.2018.0184

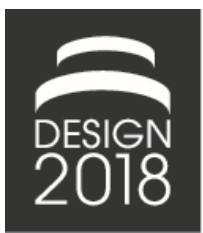

\title{
SETTING THE SCENE: A NEW PROPOSAL FOR VISUALIZING AND ESTABLISHING 3-DIMENSIONAL PERSONAS AS A MANAGEMENT TOOL
}

\author{
A. Koscher and S. Dittenberger
}

\begin{abstract}
Experiences drawn from former projects indicate a gap concerning the creation and the perpetual use of Personas throughout a project development process. This gap builds the focus of this paper. In order to develop a new approach to create a possibility for all team members to actively get involved in the Persona creation and further evolvement over the project duration, this paper offers a new option for using Personas dynamically for communication and visualization of product development stages.
\end{abstract}

Keywords: user centred design, personas, product development, project management, visualisation

\section{Introduction}

A user requirements study, applying different methods, usually takes place at the very beginning of every research project. User centred design, the focus on the wishes and skill of the actual end-user and target groups, is an essential part in AAL (active and assisted living) research projects. Although the use and the effectiveness of conducting an end-user study in a product development process seems no longer to be in question, the implementation of the gathered research findings throughout the subsequent project development stages still is. The creation of Personas, based on the findings of user requirements studies, is an already well probed method to address the need for a lasting impact of research findings on the following design phases.

However, during the work on two AAL projects the difficulty of keeping Personas vivid and alive throughout the entire product development cycle clearly came out. The need for keeping a steady focus on the data gathered during a requirements study phase, by qualitative social research and design research methods, could not sufficiently be answered by the implementation of 2-dimensional Personas. In the course of the product development process, technical aspects of the possible product and system solution got discussed primarily and the developed Personas faded into the background and got neglected. Thus, the product development tended rather towards a technique-oriented instead of a useror persona-oriented direction.

This awareness raised the questions: How can we enrich the presentation of Personas to gather a more holistic view of their lives? How can we visualize the created Personas to keep them alive and memorable during the entire project development process for the whole project team? And thus, how can we integrate the permanent preoccupation with Personas as a management tool?

In this paper we examine what kind of Personas would support best the communication amongst the project team throughout the entire product development process. We present a new approach of visualizing the findings of user requirements studies in form of 3-dimensional Personas, which includes 
tangible and emotional aspects and possesses the power to be memorized by the project team members during the whole development process in order to design real user-centred products. The goal is to develop a visual communication model, aiming at establishing a permanent user-centred communication with the visual appliance and support of Personas, where not only information about the Persona characters is presented to the team, but the team constantly has to work on and develop the Personas and their surroundings further.

\section{State of the art of Personas}

\subsection{Personas' origin}

The word persona is derived from the Latin persona, referring to the masks worn by actors in ancient theatres (Blomkvist, 2006). It was Alan Cooper who first came up with the construct of a Persona in the context of user-centred design in his publication The Inmates are Running the Asylum, defining those as "fictitious description of users (Cooper, 1999)". Cooper describes a Persona as a life-like character representing user behaviour patterns, goals and personal attitudes and motivations, applied for supporting the project team members' understanding of the target user. Applying Personas in a development process facilitates effective communication about users and reduces necessary changes at the end of the process. Grudin and Pruitt (2002) broaden the benefits and mention the possibility for extrapolation from partial knowledge of users to diverse contexts. Pruitt and Adlin (2006) stress the Personas' potential to increase the engagement of team members and argue that Personas lead to better design solutions.

To date the benefits are well-reported and well-known, still the question remains, how Personas work. Cooper (1999) argues that "[t]he more specific the persona is, the more effective they are as design tools. With more specific, idiosyncratic details, the persona becomes a »real« person in the minds of the developers." He emphasises that a Persona is a tool for communication and design within the group of designers, software developers, managers, customers and other stakeholders. Pruitt and Grudin (2003) confirm that without Personas, development teams routinely make decisions about features and implementation without recognizing or communicating their underlying assumptions about who will use the product and how it will be used. The method helps establish who is and consequently who is not being designed for, as Personas explicitly do not cover every conceivable user.

The illustration and presentation of Personas, such as text documents, posters, foldable persona cards, paper cut-outs or movies (Saez and Garreta-Domingo, 2011; Dittenberger and Koscher, 2017) generally are supposed to be beneficial for the development team, irrespective of direct contact with users or not.

\subsection{Personas, requirements, scenarios and context}

Data for the building of Personas can derive from ethnographies, market research, requirements and usability studies, interviews, observations, and other research methods. In contrast to this, Schneidewind et al. (2012) follow a different approach by defining Personas before the elicitation of requirements. Nielsen (2004) on the contrary situates her approach at the beginning of the design process after ethnographic studies or user inquiries and before system development begins. Nielsen uses the term "engaging persona" which she understands as a description of a user in a scenario. This user's description is based on field studies. Depending on goals and the situation of use, the engaging persona can be the user in one or more scenarios. The scenario focuses on a user using the system with the objective to explore design solutions. Both scenarios and engaging personas are part of narrative structures that aim at developing a memory aid.

In case that ethnographic studies are not always appropriate or possible, time consuming and expensive, other means of approximations to end user requirements is to use existing user datasets (Marshall et al., 2015).

Pruitt and Grudin (2003) outline the psychological theory why Personas are more engaging than scenarios and why it is better to develop Personas before scenarios. They argue that based on the Theory of Mind deriving from psychology, which describes the ability to predict or assume other's 
behaviours, needs, expectations and intentions, humans constantly use partial knowledge to draw inferences, make predictions and form expectations about the people around us. This human capability is brought to the design process by Personas. If Personas are well-crafted, they are generative and if team members are familiar with these Personas they can extrapolate from a situation to its affection on behaviour of this Persona and the Persona can easily be projected into new situations. A scenario on the contrary "just covers what it covers (Pruitt and Grudin, 2003)". According to John Carroll (2000) a scenario is a concrete story about use in the context of software development. Scenarios are a vocabulary for coordinating the central tasks of system development, e.g.: "A person turned on a computer; the screen displayed a button labelled Start; the person used the mouse to select the button."

Contrary to this description of a set of tasks the user performs, in planning, scenarios are seen as processes with the assumption that there are no right answers and the future is not to predict but can be discussed. Scenarios make discussions possible and make it possible for the decision makers to take strategic decisions and to work with and discuss risks (Heijden, 1996). Clausen (2000) makes a distinction between stories and scenarios where the scenario implicates a context and its role is to influence the context. The story supports the system designer's dialogue with other design groups about the design. However, in most perceptions the scenario has the ability to function as a support for communication too (Nielsen, 2004). An important aspect when creating Personas and developing them further is to keep in mind their life context which we understand in this paper according to Roto et al. (2011) as environment wherein the user experience (UX) emerges. In the context of UX, which studies the relation between the user and the system, researchers take the user and the system into focus and consider the surrounding circumstances as context (e.g. Hassenzahl and Tractinsky, 2006). Context in this regard is always seen from the user's perspective, whereas e.g. context-aware systems approach context from the system's perspective (Roto et al., 2011).

Roto et al. (2011) also underline that visiting the study contexts in advance is a crucial step of field studies. The researchers should at least have some idea of the variety of potential contexts of use and get familiar with those contexts.

\subsection{Appliance of Personas to date}

When talking about user centred and participatory design approaches of a product development process, the user requirements exploration represents the indispensable step at the beginning of the development process and subsequently within its iterations. To inform the design and development process, it is important to capture user information and feedback ideally at every stage, with input from everyone involved: users, designers and stakeholders. It's the attempt to better understand and involve real users, and is as imperative and important in creating more appropriate and user friendly products or services (Muller, 2002; Lindgaard et al., 2006).

Marshall et al. (2015) refer to a study by Goodman-Dean et al. (2010) who confirm that designers value design information that is quick and easy to use, visually stimulating, flexible, open ended and clearly and concretely related to design issues. Thus Personas suit informal and flexible working and when visually compelling are ideally suited to engaging designers and fostering empathy with user needs. Goodman-Dean et al. (2010) however warn that, as Personas focus on archetypal users, this might make it hard to communicate the range of abilities within a population. Thus more specific data about the capabilities of users, correspondent to the movement from requirements and concept generation stage to the product development stage may be needed to supplement the use of Personas.

In literature there is no doubt that Personas are a medium for communication within a project team (e.g. Cooper, 1999; Pruitt and Grudin, 2003), but a recent study by Matthews et al. (2012) revealed that most designers do not use Personas significantly in their own design work. Schneidewind et al. (2012) express the hypothesis, that Personas are mainly used on the one hand to describe the whole way of interacting with the new product from the viewpoint of the Persona, as it is crucial to communicate the conceptual model to all involved project members. And second, Personas are used by illustrators enabling them to create pictures or stories consisting of the Persona interacting with the new product.

Thus, there seems to be a gap between the use of Personas in theory and practice or in other words there is a missing link between the creation and communication of Personas - designed in a two- or 
three-dimensional form - and their applied use as a method in each step of the product development process.

Concluding, the challenge seems not to lie in the assembling of user characteristics and defining those as Personas, but in the creation of Personas that can be applied most effectively as design and communication tools. In other words, the power of Personas is limited unless they can be situated within rich contexts and are brought to life so that developers can explore how situations appear and feel from different perspectives (Lewis and Cole-Kemp, 2014). Table 1 is based on Miaskiewicz and Kozar (2011) and describes Persona characteristics defined by selected Personas authors.

Table 1. Characteristics of Persona use (based on Miaskiewicz and Kozar, 2011)

\begin{tabular}{|c|c|}
\hline Source & Specified Characteristica \\
\hline Cooper (1999) & $\begin{array}{l}\text { - Increase focus on the users and their goals } \\
\text { - Facilitate effective communication about users } \\
\text { - Reduce changes at the end of the development process }\end{array}$ \\
\hline Cooper and Reimann (2002) & $\begin{array}{l}\text { - Build consensus and commitment to design } \\
\text { - Help to measure a design's effectiveness } \\
\text { - Define the product's feature set } \\
\text { - Facilitate effective communication within the project team } \\
\text { - Help other related efforts such as marketing plans }\end{array}$ \\
\hline Grudin and Pruitt (2002) & $\begin{array}{l}\text { - Facilitate a focus on users and work contexts } \\
\text { - Make assumptions about users explicit } \\
\text { - Facilitate effective communication about the users } \\
\text { - Increase focus on a specific audience }\end{array}$ \\
\hline Nielsen (2004) & $\begin{array}{l}\text { - "Engaging Persona" as user in one or more scenarios } \\
\text { - Facilitate the exploration of design solutions } \\
\text { - Memory aid for developers }\end{array}$ \\
\hline Pruitt and Adlin (2006) & $\begin{array}{l}\text { - Narrow the users being designed for } \\
\text { - Lead to better design decisions } \\
\text { - Increase engagement among the design team } \\
\text { - Build empathy for the users }\end{array}$ \\
\hline Long (2009) & $\begin{array}{l}\text { - Strengthen focus on the users during development process } \\
\text { - Lead to more user-friendly designs } \\
\text { - Make the user needs more explicit } \\
\text { - Guide decision making }\end{array}$ \\
\hline Matthews et al. (2012) & $\begin{array}{l}\text { - Tool for communication to others } \\
\text { - Support building for a chosen design } \\
\text { - Advocate user needs }\end{array}$ \\
\hline Schneidewind et al. (2012) & $\begin{array}{l}\text { - Support concept description to team members } \\
\text { - Support description of a new product in use }\end{array}$ \\
\hline
\end{tabular}

\section{Visualisation techniques of Personas}

Once the user requirements are gathered, the question remains how and in which manner the defined requirements could be transferred to the technical product development team in order to create a lasting impact on the development process.

"Vision trumps all senses" states Susan Weinschenk (2011), meaning that visuals are easier to remember as words or sounds. People react strongly especially to faces and faces looking right at people will have an emotional impact. To memorize, humans need to hear information over and over. People use schemata to store information in long-term memory and to retrieve it. Schemata are combined information of different parts, which makes them easier to remember (e.g. the parts of a head like eyes, ears, nose, mouth etc. are part of a head). If people can connect new information to 
already stored information, it is easier to make it stick or stay in long-term memory, and it is easier to retrieve it. (Weinschenk, 2011) Obviously the task of a researcher includes the clear and vivid visualisation of information to not only make the findings visible in general but furthermore to memorize them for a longer period of time, e.g. for the whole process of a product's development. As we know from neuroscience, data gets an easier access to long term memory if its perception is connected to emotional as well as tangible aspects. Moreover, data is better perceived and memorized if there is the possibility to experience it with more than one sense, e.g. if visual, haptic and acoustic stimuli are offered.

There is ongoing research on how to stimulate developers' interest in Personas for internalizing key characteristics of the users for whom a product is going to be designed via specified visualisation techniques. There is research in how to visualise data in general in two-dimensional ways (Pruitt and Grudin, 2003; Nieters et al., 2007; Hunter, 2012; Lewis and Coles-Kemp, 2014) and in threedimensional ways (Saez and Garreta-Domingo, 2011; Dittenberger and Koscher, 2017), always addressing the question how to get product teams connected to specific characteristics of users. For example, Nieters et al. (2007) describe the production of physical Personas in form of cards in order to stimulate interest, create fun, stickiness and confidence in persona content for product teams. Personas were presented in form of action figures, which were displayed on posters and pop-ups. Nieters et al. (2007) report that this solution turned out to be memorable for the developers and it instilled confidence in the Persona content.

The visualisation of three-dimensional Personas instead is less disseminated, yet there exist examples of attempts to create more tangible and interactive Personas presentations. Saez and Garreta-Domingo (2011) combined the concept of Personas and scenarios and created a playful and more permanent artefact to hold the information, which should foster the understanding of users by showing their main contexts, as contexts are becoming more important with the use of mobile devices. Contrary to Weinschenk (2011) who underlines the importance of faces concerning memory, Saez and GarretaDomingo (2011) chose to reduce the bias that a face can generate, using silhouettes instead.

The foldable Personas card presents a new way of a visualization technique for Personas with the goal not only to display information but to translate this information into a tangible, fun creating and curiosity awakened experience (Dittenberger and Koscher, 2017). These Persona cards present the most significant findings for two different user groups with regards to their family, living, health and care situation, their social activities and preferred leisure time activities, their communication habits and technology usage behaviour and finally their wishes and aspirations concerning a relaxed care situation. Each foldable card is designed to animate the project team members to actively get in contact with the Persons of the target group. Each page of the foldable card presents another aspect of the created Persona. Additionally, the social connection between the two user groups are included in this new visualization method. So for the user of the foldable Persona cards not only information can be gained by interacting with it but also moments of surprise which aspect of the life of the user will be presented in the next page. Table 2 shows an overview of exemplary two- and three-dimensional Persona visualisation.

Comparing two- to three-dimensional Personas visualisation we follow arguments from neuroscience that the more senses are involved the better information is memorized. The authors try to prove this assumption in the course of an ongoing AAL-project by comparing the outcome to those of a former AAL-project. 
Table 2. Overview of two- and three-dimensional Personas visualisation

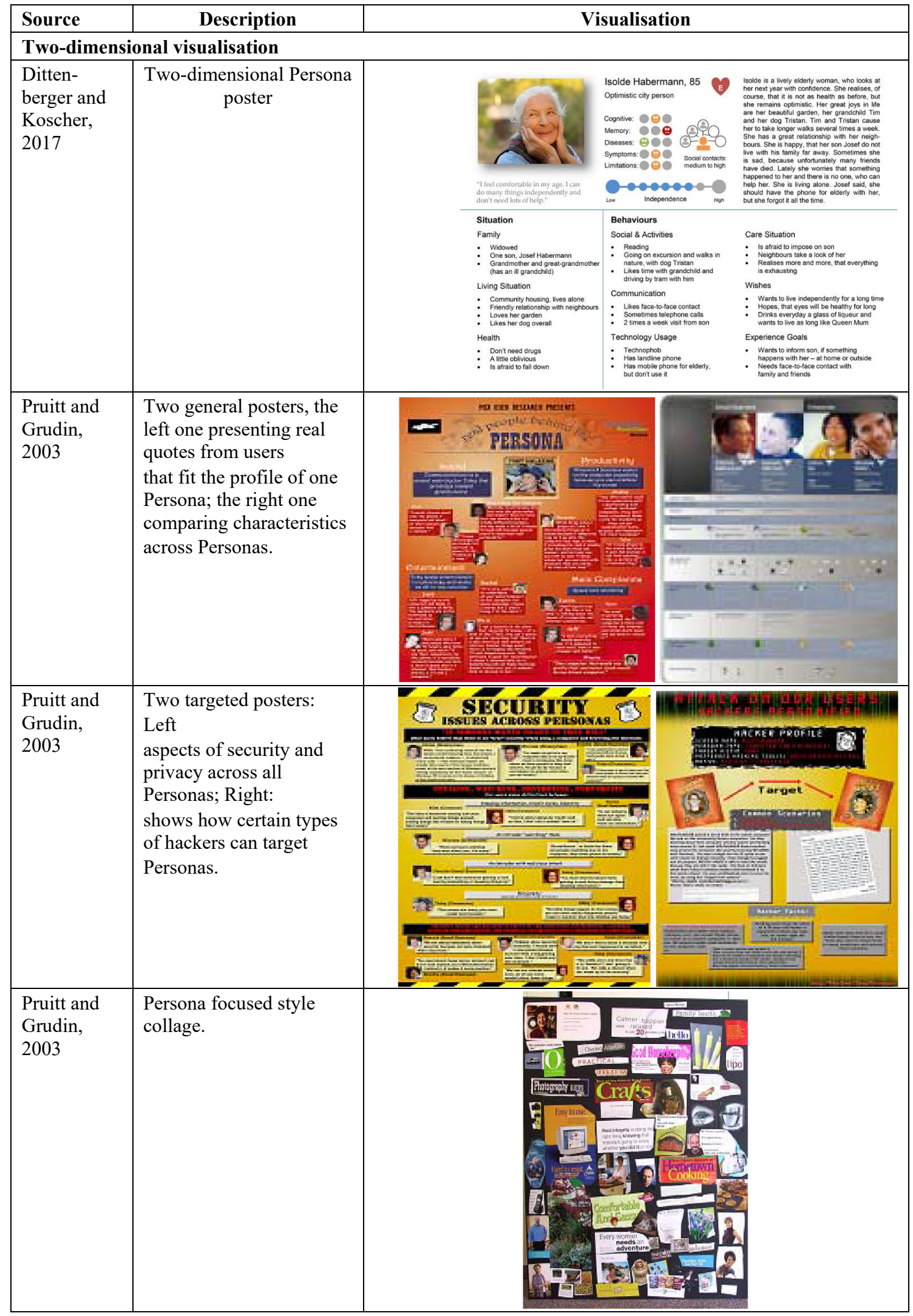




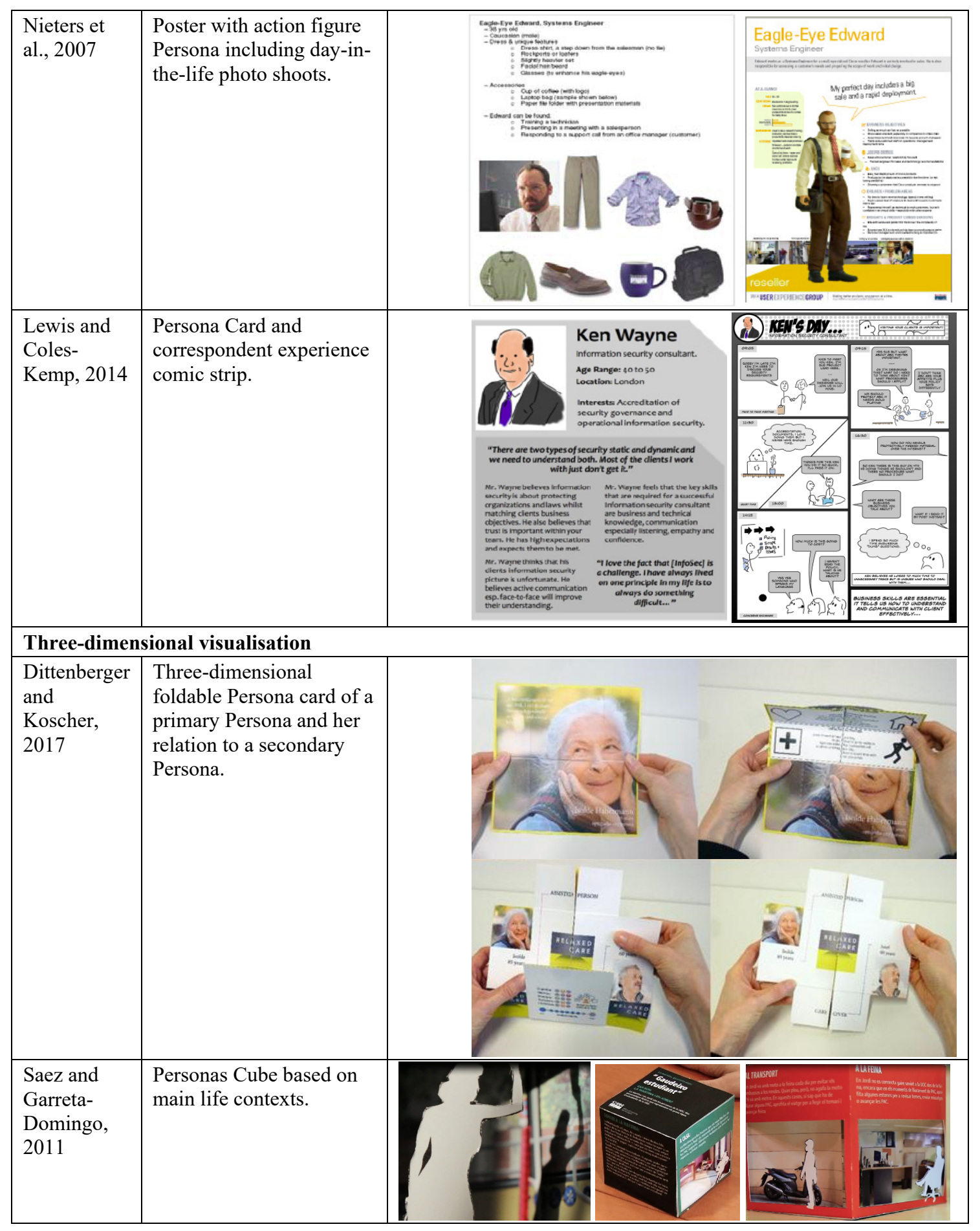

\section{Discussion: Personas as means of visualisation of research findings and communication tool amongst project team members}

As confirmed by Nielsen (2004) there is a permanent need to include Personas in the project development process, but still there is the open question how to provide or establish a permanent communication process including well-visualized Personas. 
Matthews et al. (2012) claim that it is particularly important to consider how Personas are presented to practitioners and suggest an approach which presents user information to designers in three easily separable layers: Persona, user role and user study data, based on the question how Personas can coexist with a continuous need for immersion in user studies.

Nielsen (2004) suggests a structured process starting with discussions among the team members of whom to target and create written Personas. Thus the creation of a shared understanding of the core user is supported by creating opportunities to constantly refine and broaden the understanding of the user in relation to context in terms of surroundings, situations, and needs. Nielsen's (2004) proposed model is a tool for communication and it shifts the focus to the process of understanding the user and exploring the design area (Figure 1).

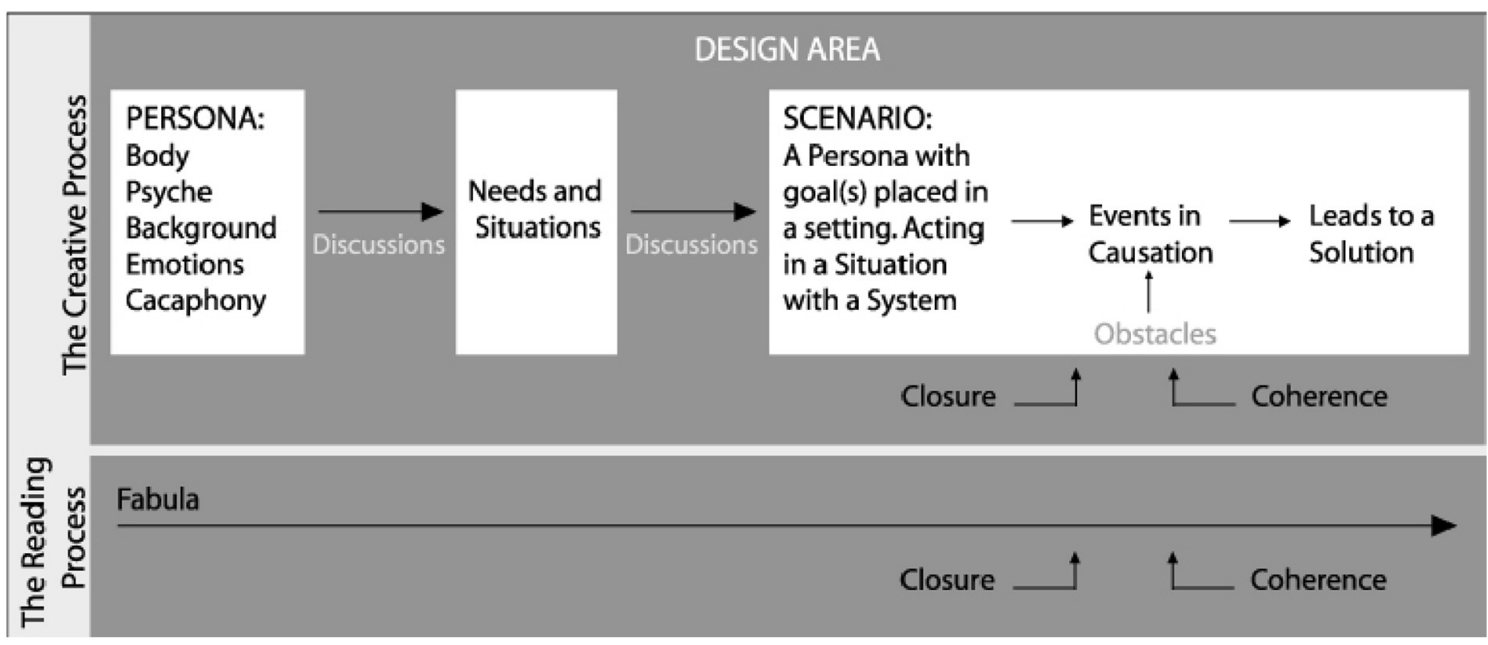

Figure 1. Process of perceiving Personas and scenarios (Nielsen, 2004)

The model with the three steps engaging Persona, needs and situation, and narrative scenarios offer room for specific discussions that are focussed on the user. Those discussions widen the knowledge about the user, reveal stereotypes and inferences and offer opportunities for adjustment. It therefore meets the need of a user-centred permanent communication among the project team members. Yet there is still the open question how to establish well-crafted visualised Personas to a permanent communication culture, as also Schneidewind et al. (2012) hypothesize the use of Personas at the end of the development process as a mean to describe or illustrate the new product. This "fading-out" phenomenon of Personas during the project development process corresponds with our experience in AAL projects, where Personas played their role strongly in the beginning of the project, and then again in the end when it came to explaining and communicating a product, but they got neglected during the designing phase, especially by technical partners. Although we uncovered relevant factors for assessing user characteristics at an early stage of the project to ensure a profound output as a basis for the subsequent project development process, the in this way steadily enriched Personas did not play the important role they should play.

Consequently, the aim of our approach is the entanglement and the merge of the two areas, communication and visualisation of Personas during the entire product development process.

Based on Nielsen's (2004) and Schneidewind et al.'s (2012) approach we suggest to advance those theories further and develop a visual communication model, aiming at establishing a permanent usercentred communication with the visual appliance and support of Personas, where not only information about the Persona characters is send to the team, but the team constantly has to actively work on and develop the Personas and their surroundings further.

In a first step when introducing user-studies based Personas to the team we want to provide the project team with visuals of the following components: Persona of our target group, context/surroundings and speech bubbles (Figure 2). As long as research findings for the creation of Personas are still to be gathered, this process might also start with the common creation of Assumption Personas to get the team into the topic. 


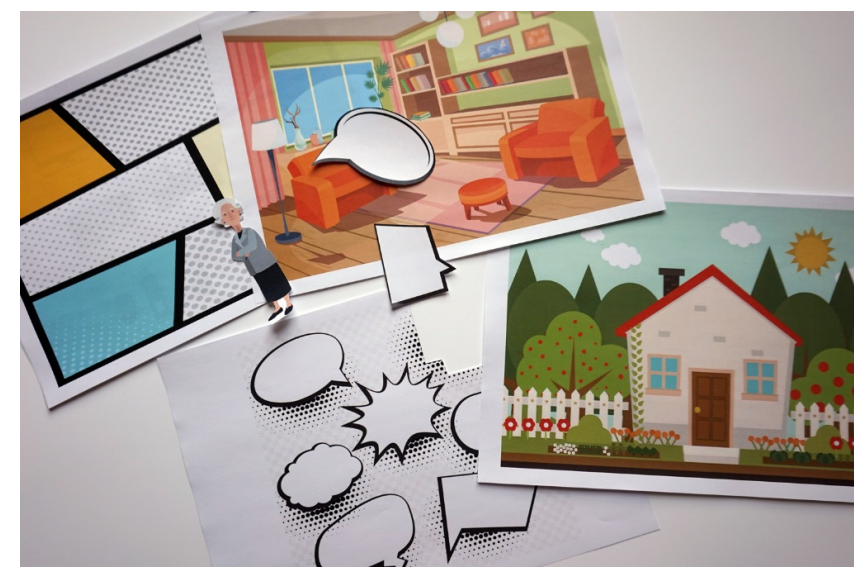

Figure 2. Components for the first persona communication meeting

During every meeting the Persona is commonly developed further and enriched with personal statements expressing her current status, anxieties, wishes, hopes, needs etc. (Figure 3 and Figure 4).

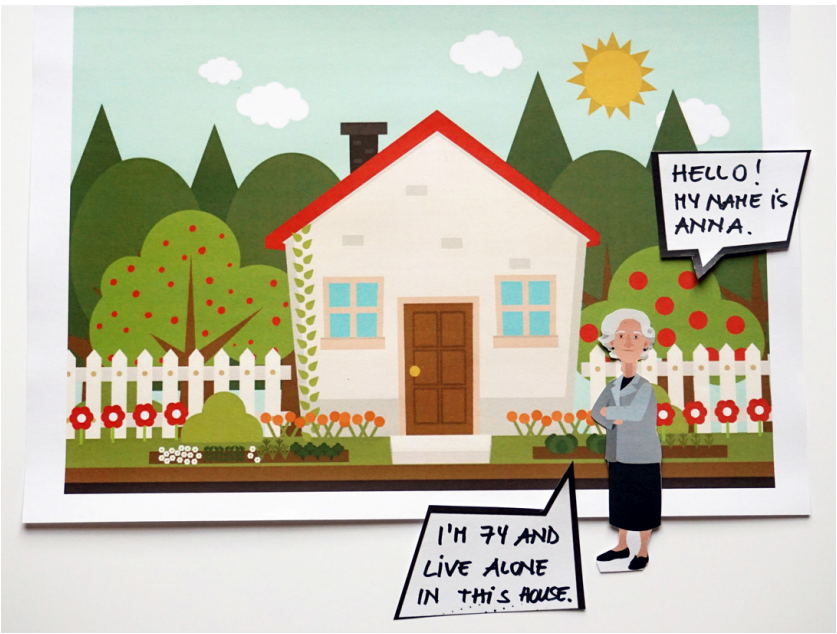

Figure 3. Combining Persona, context and surrounding

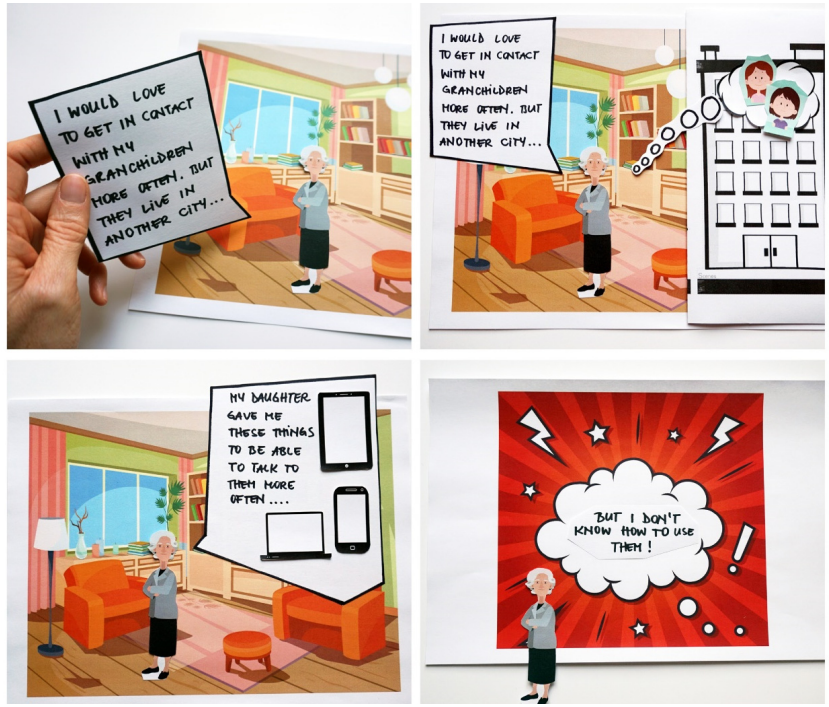

Figure 4. Visual Persona development in the course of the project 
Step by step the Persona becomes more familiar to the project development team members. This approach aims at supporting a permanent companionship of Personas during product development, starting with the common creation of project Personas (Figure 5), moving on to the development of context and surroundings and leading them to a permanent iterative interaction with possible product developments. Personas shall no longer be experienced as a method only, but as an integrated part of project management.

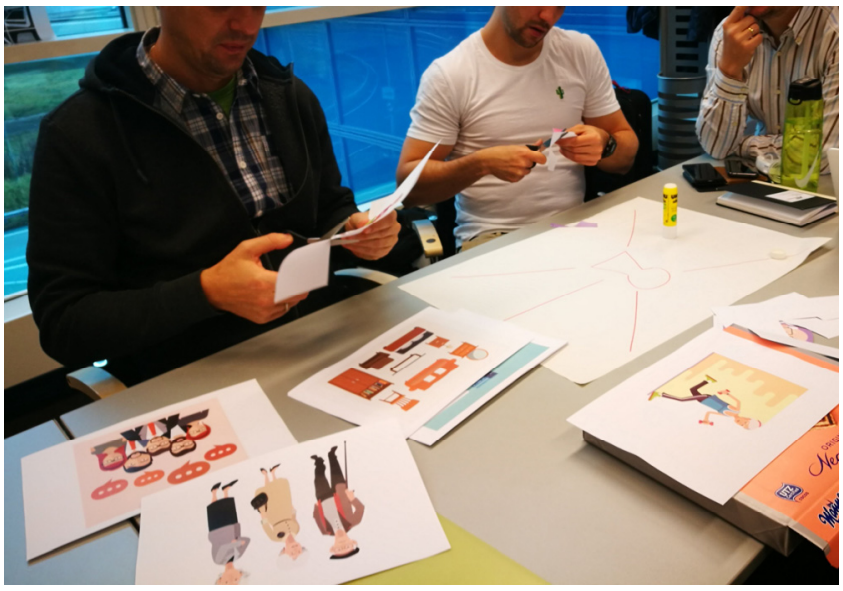

Figure 5. Technical project team members during the creation of project-linked visual Personas

\section{Conclusion}

We are well aware that the focus of this paper lies not in the formulation of a new theory on Personas. Our aim was to provide an overview of how Personas were applied in different projects right from the beginning of being defined. Based on our experience which pinpointed the limitations of the appliance and the feasibility of Personas, we want to address the problem of constant Persona communication, supported by the hypothesis of Schneidewind et al. (2012) who depicted the predominant use of Personas for mainly descriptions of product interaction after the product development process.

We therefore suggest to frame the Personas method as a management tool right from the beginning of a product development process. Considered as a management tool combined with communication, the permanent engagement of the whole project team with Personas has a more obligatory character and will lead to a constant and strong presence of the target user during development phases.

A further aspect is the implementation of an adequate visualisation method which answers the question what kind of Personas would support best the communication amongst the project team throughout the entire product development process. As mentioned earlier knowledge from neuroscience points to the aspect that the more senses are involved the better information is memorized. Our approach is based on this data and aims to create 3-dimensional Personas which create a deeper impact. The authors try to prove this assumption in the course of an ongoing AAL-project by comparing the outcomes to those of a former AAL-project. In the context of establishing the Personas method as a management tool other aspects such as the permanent repetition of information and the permanent engagement with Personas have to be examined as well.

With reference to the initial questions concerning the enrichment of Personas to a more holistic view of their lives, the visualization of Personas to keep them alive and memorable during the entire project development process for the whole project team, and finally the implementation of the Personas method as a management tool to foster permanent preoccupation with Personas, we feel encouraged to move on with the approach to support the appliance of 3-dimensional Personas including multi-sensory stimuli as well as emotional aspects. Still there is the need to insist on the permanent preoccupation with Personas by all project team members, which in our approach might be played out by establishing Personas as a management tool. This approach is currently empirically researched in an ongoing AAL product development project. 
For an even more intensive visual impact we consider to enlarge the current Persona paper scenes to 3dimensional theatre stage sceneries. The current proposal has the advantage of being mobile and therefore easily transportable in order to apply it as management method for visualising the conclusions of each team meeting in form of a Persona scene. The sceneries are easily duplicated and rebuilt.

Our further work will pursue the internal campaigning of Personas within AAL-projects by following the developed approach. A survey amongst the project team members at the final stage of each project will reveal further insights into the perception of this approach on behalf of the project team members.

\section{Acknowledgements}

AAL-projects are co-funded by the AAL Joint Programme and the National Authorities and R\&D programs of the participating countries.

\section{References}

Blomkvist, S. (2006), The user as a personality: A reflection on the theoretical and practical use of Personas in HCI design. [online] Uppsala University, Sweden. Available at: https://www.it.uu.se/research/publications/reports/2006-049/2006-049-nc.pdf (accessed 04.12.2017)

Carroll, J. (2000), Making use: Scenario-based design of human-computer interactions, MIT Press. https://doi.org/10.1145/347642.347652

Clausen, H. (2000), Informationsteknologiens menneskelige grundlag, Teknisk Forlag, København.

Cooper, A. (1999), The Inmates are Running the Asylum, Macmillan Publishing Co., Indianapolis, IN, USA. https://doi.org/10.1007/978-3-322-99786-9_1

Dittenberger, S. and Koscher, A. (2017), "Tangible information: an exploration on visualization techniques to keep a steady focus on research findings throughout a design project development process", Proceedings of the Cumulus REDO Conference 2017, Kolding, DK, May 30 - June 2, 2017, Cumulus International Association, Aalto.

Grudin, J. and Pruitt, J. (2002), "Personas, participatory design and product development: An infrastructure for engagement", Proceedings of the participatory design conference, Malmö, Sweden, June, 2002, ACM Press, pp. 144-161.

Hassenzahl, M. and Tractinsky, N. (2006), "User Experience - a research agenda (Editorial)", Behavior and Information Technology, Vol. 25 No. 2, pp. 91-97.

Heijden, K. (1996), Scenarios - The Art of Strategic Conversation, John Wiley and Sons, Chichester.

Hunter, W. (2012), Data Insights: New Ways to Visualise and make sense of Data, Morgan Kaufmann Publishers Inc.

Lewis, M. and Coles-Kemp, L. (2014), "Who Says Personas Can't Dance? The Use Of Comic Strips To Design Information Security Personas", CHI 2014, Toronto, ON, Canada, April 26-May 01, 2014, ACM Press, pp. 2485-2490. https://doi.org/10.1045/2559206.2581323

Lindgaard, G., Dillon, R., Trbovich, P., White, R., Fernandes, G. and Lundah, S. (2006), "User Needs analysis and requirement engineering: theory and practice", Interacting with Computers, Vol. 18 No. 1, pp. 47-70. https://doi.org/10.1016/j.intcom.2005.06.003

Long, F. (2009), "Real or Imaginary; The Effectiveness of Using Personas in Product Design", Proceedings of the Irish Ergonomics Society Annual Conference, Dublin, May 2009, Irish Ergonomics Society, pp. 1-10.

Marshall, R., Cook, S., Mitchell, V., Summerskill, S., Haines, V. et al. (2015), "Design and Evaluation: End Users, User Datasets and Personas", Applied Ergonomics, Vol. 46, pp. 311-317. https://doi.org/10.1016/j.apergo.2013.03.008

Matthews, T., Judge, T.K. and Whittaker, S. (2012), "How Do Designer and User Experience Professionals Actually Perceive and Use Personas?", CHI 2012, Austin, Texas, USA, May 5-10, 2012, ACM Press, pp. 12191228. https://doi.org/10.1145/2207676.2208573978-1-4503-1015-4/12/05

Miaskiewicz, T. and Kozar, K. (2011), "Personas and user-centred design: How can personas benefit product design processes?”, Design Studies, Vol. 32 No. 5, pp. 417-430. https://doi.org/10.1016/j.destud.2011.03.003

Muller, M. (2002), "Participatory Design: the third space in HCI", In: Jacko, J. and Sears, A. (Eds.), The human computer interaction handbook: Fundamentals, evolving technologies and emerging applications, Lawrence Erlbaum, Mahwah, NF, USA.

Nielsen, L. (2004), Engaging Personas and Narrative Scenarios - a study on how a user-centred approach influenced the perception of the design process in the e-business group at AstraZeneca, $\mathrm{PhD}$ thesis, Copenhagen Business School, Denmark.

Nieters, J., Ivaturi, S. and Ahmed, I. (2007), “Making Personas memorable”, CHI 2007, San Jose, California, USA April 28 - May 3, 2007, ACM Press, pp. 1817-1823. https://doi.org/10.1145/1240866.1240905 
Pruitt, J. and Adlin, T. (2006), The persona lifecycle: Keeping people in mind throughout product design, Morgan Kaufmann, San Francisco.

Pruitt, J. and Grudin, J. (2003), "Personas: Practice and Theory", Proceedings of the 2003 Conference on Designing for user experiences, San Francisco, California, June 6-7, 2003, ACM Press, pp. 1-15. https://doi.org/10.1145/997078.997089

Roto, V., Väätäjä, H., Jumisko-Pyykkö, S. and Väänänen-Vainio-Mattila, K. (2011), "Best Practices for Capturing Context in User Experience Studies in the Wild", Proceedings of the 15th International Academic MindTrek Conference: Envisioning Future Media Environments, Tampere, Finland, September 28-30, 2011, ACM Press, pp. 91-98. https://doi.org/10.1145/2181037.2181054

Saez, A.V. and Garreta-Domingo, M. (2011), "Scenario-based Persona: Introducing Personas through Their Main Context", CHI 2011, Vancouver, BC, Canada, May 7-12, 2011, ACM Press. https://doi.org/10.1145/1979742.1979563

Schneidewind, L., Hörold, S., Mayas, C., Krömker, H., Falke, S. and Pucklitsch, T. (2012), "How Personas Support Requirements Engineering", Proceedings of the First International Workshop on Usability and Accessibility Focused Requirements Engineering, Zurich, Switzerland, June 4, 2012, IEEE Press, pp. 1-5.

Weinschenk, S.M. (2011), 100 things every designer needs to know about people, New Riders, Berkeley, California.

Andrea Koscher, Mag., MDes / Senior Researcher

New Design University St. Pölten, Design

Mariazellerstr. 97a, 3100 St. Pölten, Austria

Email: andrea.koscher@ndu.ac.at 